\title{
Development and Comparison of Symptom Indices for Quantifying Grapevine Resistance to Pierce's Disease
}

\author{
A. F. Krivanek, J. F. Stevenson, and M. A. Walker
}

First and third authors: Department of Viticulture and Enology, One Shields Ave., University of California, Davis 95616; and second author: Department of Biology and Chemistry, Texas A\&M International University, 5201 University Blvd., Laredo 78041. Accepted for publication 14 September 2004.

\begin{abstract}
Krivanek, A. F., Stevenson, J. F., and Walker, M. A. 2005. Development and comparison of symptom indices for quantifying grapevine resistance to Pierce's disease. Phytopathology 95:36-43.

Symptoms of Pierce's disease (PD) were assessed under greenhouse conditions on field-resistant and field-susceptible grapevines in order to characterize the PD resistance phenotype in the genus Vitis. A cane maturation index (CMI) was developed to quantify the green-islands symptom, which was measured at 12 weeks post-bacterial inoculation, along with leaf scorch and percentage of xylem vessels blocked by occlusions. Canes of resistant genotypes matured normally and had a significantly lower CMI score of 0.9 (on a 0-to-6 scale) compared with 5.1 for the susceptible genotypes. The CMI scoring method had a high correlation $\left(R^{2}=0.91\right)$ with previously characterized field performance, whereas leaf scorch had only a moderate correlation $\left(R^{2}=0.51\right)$ with field performance. Average scorched area on leaves of the susceptible and resistant
\end{abstract}

ABSTRACT genotypes was 80 and $48 \%$, respectively, demonstrating that leaf scorch can be extensive in resistant genotypes under the presented screening conditions, and suggesting that systemic infection can occur in all evaluated genotypes. Occlusions within both stem and petiole vessels were composed principally of tyloses and were significantly higher in petioles than in stems of either resistant or susceptible backgrounds. Susceptible genotypes displayed a higher level of stem tylose occlusions relative to resistant genotypes, but correlation to field performance was low $\left(R^{2}=\right.$ 0.13 ). Ease of use and high correlation to field performance makes CMI scoring a better choice for PD resistance evaluations relative to other phenotypic symptom assessments.

Additional keywords: Blanc Du Bois, Chardonnay, Chenin blanc, D'Arpa, D8909-15, Roucaneuf, Tampa, V. aestivalis subsp. smalliana, V. arizonica, V. shutttleworthii, V. simpsonii, V. vinifera, Xylella fastidiosa, Zehnder 71-50-1.
Pierce's disease (PD) of grapevines is caused by the bacterium Xylella fastidiosa and is the primary restriction to grape cultivation in many areas of southern North America. The pathogen is vectored to grape plants primarily by xylem-feeding sharpshooter insects $(17,19)$ from a wide range of host plant species $(7)$. Management strategies to reduce vector populations and remove host plants can have negative environmental impacts and have proven only marginally effective in lessening the severity of PD within areas of intense disease pressure. An alternative control strategy is the development of PD-resistant grape cultivars, which requires accurate and efficient assessment of disease advancement in a grapevine.

A characteristic symptom of PD is leaf scorching, with zones of chlorosis progressing into necrotic zones at the peripheral margins of infected leaves. Other common symptoms include early defoliation, fruit cluster dehydration, dwarfed shoots, uneven maturation of cork on infected canes, cane stunting, dieback, and eventual plant death $(10,11,17)$. The uneven cane maturation symptom has been referred to as "green islands" $(3,6,13)$. These green patches on the surface of the cane are a result of incomplete development of the cork cambium or phellogen. In healthy plants, the phellogen forms a complete ring around the cane at the depth of the primary phloem. An active phellogen produces cork toward the outside and phelloderm toward the inside, and these layers collectively compose the periderm (31). In healthy grapevines, formation of the periderm isolates the epidermis, cortex, and primary phloem from the secondary phloem-conducting system,

Corresponding author: M. A. Walker; E-mail address: awalker@ucdavis.edu

DOI: 10.1094/PHYTO-95-0036

(C) 2005 The American Phytopathological Society causing these tissues to die and form the uniform brown covering of a mature cane. In diseased grapevines, the phellogen formation is patchy, resulting in the retention of the immature green islands on the cane surface.

Although PD symptoms in grapevines have been well described, researchers have relied primarily on longevity studies to evaluate grape germ plasm for $X$. fastidiosa resistance. One study conducted in Mississippi evaluated an extensive collection of grape species from the southern United States over a 25-year period, and based resistance on longevity, vigor, and general PD symptoms (24). Other studies have used longevity evaluations along with disease indexing to screen for resistant genotypes (18, 25,32). However, a typical PD field evaluation based on grapevine survival takes 5 to 25 years, making it impractical for breeding and genetic analysis research.

Procedures other than longevity studies for symptom quantification under field conditions have been utilized. In citrus, an index based on percent area of chlorotic leaves was developed to evaluate citrus variegated chlorosis (CVC) severity on trees infected with a CVC $X$. fastidiosa pathotype (1). In grape, an index based on percentage of scorched leaves was used to assess progression of $X$. fastidios $a$ growth and internal vascular plugging in a field-evaluated Muscadinia rotundifolia (muscadine) grape cultivar (26). Many of the typical external disease symptoms associated with PD often are correlated with the internal disease symptom of vascular plugging (occlusions) (17). Xylem vessel occlusions have been used to measure disease severity in resistant muscadine cultivars and in susceptible Vitis bunch grape cultivars. These studies focused on naturally infected grapevines in the field $(15,21,26,27)$, on greenhouse evaluations after artificial inoculations $(8,20)$ and in both the field and greenhouse (22). Greenhouse evaluations tend to give a more precise measurement of re- 
sistance because many of the confounding environmental and biotic effects that often are confused with external PD symptoms, such as water and salt stress, mineral deficiencies, and decline from Eutypa and Phylloxera spp. (10), can be avoided. A greenhouse evaluation that correlates well with field results would also considerably shorten the evaluation time needed for PD resistance breeding projects and genetic analyses.

The objective of this study was to quantitatively measure PD symptom development in grapevines with known field reactions so as to precisely characterize the resistance phenotype under greenhouse conditions. Resistance levels would be based on quantitative measures of leaf scorch, cane maturation, and tylose and gum occlusions in xylem vessels. We chose resistant Vitis genotypes that represent a level of diversity such that subsequent conclusions about the PD resistance reaction could be broadly applied to the Vitis genus, in contrast to previous studies which focused only on resistant $M$. rotundifolia grape cultivars. These resistant genotypes also have high-quality parents within their pedigree and are representative of PD-resistant cultivars that would be grown commercially. Finally, the selected genotypes have varying degrees of resistance and susceptibility in the field such that the degree of correlation between the field and greenhouse evaluations could be used to determine the relative effectiveness of the latter for PD resistance measurements.

\section{MATERIALS AND METHODS}

Plant material. Six field-resistant and two field-susceptible Vitis genotypes were evaluated in this study (Table 1). Blanc Du Bois was bred in Leesburg, FL, utilizing Vitis aestivalis subsp. smalliana and V. simpsonii and was described as "resistant to PD, rarely showing symptoms" $(11,28)$. Roucaneuf (SV12.309) is a complex hybrid including $V$. cinerea and $V$. berlandieri (9), and has been described as "fully resistant" to PD (30). Tampa was developed as a grape rootstock based on V. aestivalis subsp. smalliana and has been described as "resistant to PD" $(11,29)$. Zehnder 71-50-1 was bred in Summerville, SC utilizing V. aestivalis subsp. smalliana and $V$. simpsonii. It has been long lived (15+ years) with vigorous growth in Orlando, FL and Summerville, SC, both areas with high disease pressure (B. Zehnder to D. Ramming, personal communication). The selection D8909-15 was created in a cross with $V$. arizonica by H. P. Olmo at the University of California at Davis and is field resistant. D'Arpa was selected from a $V$. shutttleworthii mothervine and has been described as "moderately resistant to PD" (J. A. Mortensen to United States Department of Agriculture-Agricultural Research Service Clonal Germplasm Repository in Davis, CA, personal communication) and, as such, it is the only resistant genotype in this study having a lower level of field resistance. The two susceptible cultivars Chenin blanc and Chardonnay are pure $V$ vinifera. No $V$. vinifera is resistant to $\mathrm{PD}$ and all eventually die in the field $(11,12,19,24)$; however, their susceptibility varies. Chardonnay is one of the most susceptible and Chenin blanc is one of the least susceptible under California field conditions (32-34).
Plants were propagated from green cuttings taken from grapevines in the University of California, Davis vineyards, rooted in cellulose sponges on intermittent mist beds with $27^{\circ} \mathrm{C}$ bottom heat, and then planted in $2.5-\mathrm{cm}$ pots with a $1: 1: 1$ Yolo sandy loam soil/perlite/peat mix. Four-week-old rooted plants were transplanted to $10-\mathrm{cm}^{2}$ pots and, to ensure uniform shoot growth, cut back to two buds and regrown. Once the main shoot reached $90 \mathrm{~cm}$, lateral and apical shoot tips were removed every 3 weeks, on average, to facilitate light penetration and air circulation. To prevent $X$. fastidiosa movement into water-inoculated controls via mechanical means, shear blades were cleaned with $70 \%$ ethanol. Greenhouse temperature was maintained at 20 to $32^{\circ} \mathrm{C}$. Actively growing plants were watered for $5 \mathrm{~min}$, twice per day, using 0.5 gallon/h emitters and fertilized with a $25 \%$ Hoagland's solution (Sigma-Aldrich, St. Louis) via the drip system. The plants received, on average, $18 \mathrm{~h}$ of light per day from both ambient and supplemental lighting.

Experimental design. Four to five $X$. fastidiosa-inoculated replications were used per genotype. The individual potted plants of each genotype served as the experimental unit in the design and they were randomly distributed on greenhouse benches in an unbalanced completely randomized design. Two to four waterinoculated replicates of each genotype served as controls.

Bacteria and inoculation. An $X$. fastidiosa isolate derived from the Stags Leap area of Napa Valley, CA was used in this study and maintained in greenhouse-grown Chardonnay plants. Bacteria were isolated from infected Chardonnay plants by a microcentrifuge procedure which combines and modifies the techniques reported by Davis (5) and Berisha (2). Stem sections were surface sterilized in $1 \%$ sodium hypochlorite for $30 \mathrm{~s}$ followed by $10 \mathrm{~s}$ in $95 \%$ ethanol and briefly flamed. Sections were placed in sterile 2.5-ml Eppendorf tubes with $200 \mu \mathrm{l}$ of $\mathrm{ddH}_{2} \mathrm{O}$ and centrifuged at $16,000 \times g$ for $2 \mathrm{~min}$. The water-xylem fluid $(\approx 200 \mu \mathrm{l})$ mixture was plated onto solid periwinkle wilt medium (4) and incubated at $29^{\circ} \mathrm{C}$. The $X$. fastidiosa bacteria were maintained by streaking to fresh PW plates at 10-day intervals and reisolated from infected grapevines at 2-month intervals to insure that virulence was not lost. To prepare for inoculations, actively growing bacteria (not older than 5 days) were washed from a plate with $\mathrm{ddH}_{2} \mathrm{O}$, and the cell suspension was standardized with $\mathrm{ddH}_{2} \mathrm{O}$ to an absorbance at $600 \mathrm{~nm}$ of 0.25 , (approximately $6 \times$ $10^{8} \mathrm{CFU} / \mathrm{ml}$ as determined by culture plating). Inoculations via the pinprick procedure (14) were performed approximately 6 weeks after the grapevines had been cut back, when all plants had reached a height of 30 to $40 \mathrm{~cm}$. A position 20 to $30 \mathrm{~cm}$ above the base of each shoot was chosen as the point of inoculation (POI) for $10 \mu \mathrm{l}$ of bacterial suspension. All grapevines were inoculated above the nearest node and reinoculated 3 days later below that node to prevent escapes. Control plants were inoculated with $\mathrm{ddH}_{2} \mathrm{O}$ in a similar manner.

Disease phenotype scoring methods. Symptoms of PD were measured on $X$. fastidios $a$ and water-inoculated controls 12 weeks postinoculation. Leaf scorching was quantitatively measured by taking the mean percentage of scorched area on four leaves above

TABLE 1. Vitis genotypes used in this study and their status under field Pierce's disease (PD) pressure as reported previouslyy

\begin{tabular}{lccl}
\hline Genotype & Field PD status description & Field PD status rank & \multicolumn{1}{c}{ Species background $^{\mathrm{z}}$} \\
\hline Blanc Du Bois & Resistant & 1 & Vitis aestivalis subsp. smalliana and V. simpsonii \\
Roucaneuf & Resistant & 1 & V. cinerea and V. berlandieri \\
Tampa & Resistant & 1 & V. aestivalis subsp. smalliana \\
Zehnder 71-50-1 & Resistant & 1 & V. aestivalis subsp. smalliana and V. simpsonii \\
D8909-15 & Resistant & 1 & V. arizonica \\
D'Arpa & Resistant & 2 & V. shutttleworthii \\
Chenin blanc & Susceptible & 3 & V. vinifera \\
Chardonnay & Susceptible & 4 & V. vinifera \\
\hline
\end{tabular}

${ }^{y}$ Field PD status rank is a numerical designation of the genotype's field performance: 1, 2, 3, and 4 indicate field resistant, moderate field resistant, moderately field susceptible, and field susceptible, respectively.

${ }^{z}$ Species background description is the most probable source from which the PD resistance is derived in the resistant genotypes. 
the POI at positions approximate to $+10,+20,+30$, and $+40 \mathrm{~cm}$. Leaves completely absent at these positions were given a score of $100 \%$. A cane maturation index (CMI) rating system for PD resistance was developed by summing scores for three PD symptoms expressed on the canes at the POI to 30 to $40 \mathrm{~cm}$ above the POI. The first score estimated the percent area of cane surface not fully mature, with $0=0 \%, 1=1$ to $49 \%, 2=50$ to $99 \%$, and $3=100 \%$. The second score was based on cane tissue color; light green, yellow green, or tan canes were assigned 0 and canes with dark green tissue color were assigned 1 . The third score was an estimate of percentage of the cane that was necrotic or exhibiting dieback at the apex of the plant, where $0=0 \%, 1=1$ to $10 \%$, and $2=>10 \%$. Thus, the CMI is a composite score of disease severity with six levels, ranging from 0 for nonsymptomatic plants to 6 for the most severely affected plants. Canes with representative CMI scores are shown in Figure 1.

Sampling and microscopy methods. To quantitatively measure tylose and gum occlusions within xylem vessels, samples were taken from stem sections and petioles at 40 to $50 \mathrm{~cm}$ above the POI. Samples were dissected, fixed in formalin, glacial acetic acid, and ethanol (FAA), and dehydrated through an ethanol series (35). The samples were infiltrated with Hemo-De (Fisher Scientific, Hampton, NH) and then with Paraplast Extra paraffin (Fisher Healthcare, Houston) using a Leica TP 1020 automatic tissue processor (Leica Microsystems, Bannockburn, IL), and embedded with a Leica EG 1160 paraffin-embedding center. Samples were sectioned at $10-\mu \mathrm{m}$ thickness with a Microm HM $340 \mathrm{E}$ rotary microtome (Microm, Walldorf, Germany) and then stained following the Johansen's safranin and fast green protocol (35). Prepared tissues were observed with a compound light microscope and images were captured digitally.

Statistical analyses. Leaf scorch, CMI, and the tyloses data were evaluated in three separate analyses with the MIXED procedure of the computer program SAS (version 8; SAS Institute, Cary, NC). The experimental unit was the individual potted plant of each genotype and it was treated as a random factor. Due to the unbalanced nature of the design, the Satterwaith method was used for estimating degrees of freedom. Fixed factor treatments for the first two data sets were inoculation, field PD status (the reported field PD performance), and genotype (nested within field PD status). Field PD status had two levels (resistant genotypes as a

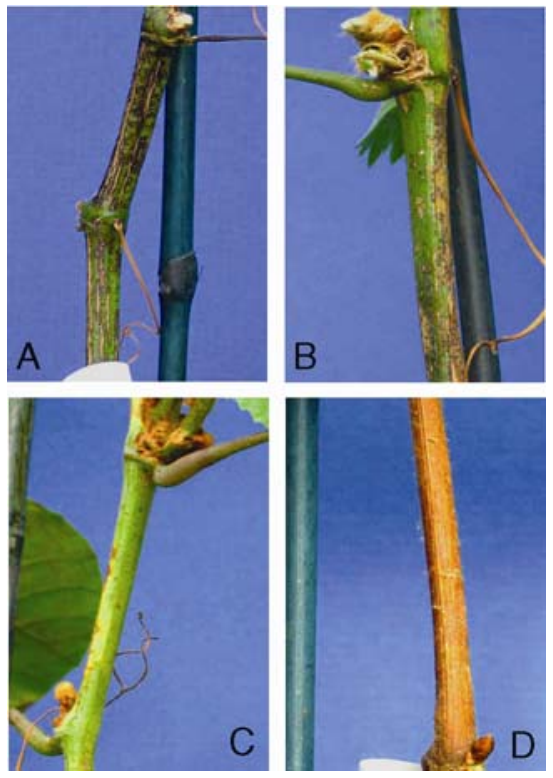

Fig. 1. Range of Pierce's disease symptoms on grapevines with varying cane maturation index (CMI) scores at 12 weeks postinoculation with Xylella fastidiosa and scored under greenhouse conditions. (clockwise from upper left: CMI scores $=6,5,3$, and 0 , respectively). group and susceptible genotypes as a group), genotype had eight levels (two susceptible and six resistant individual genotypes), and inoculation had two levels ( $X$. fastidiosa inoculated and water inoculated). The first evaluation tested the effects of the fixed factors on leaf scorch and the second analysis tested the effects of the fixed factors on CMI scores. Fixed-factor treatments for the third analysis of tylose occlusions were field PD status, genotype, and plant part. Plant part was treated as a repeated measures (within subject) factor with two levels (stem internode and petiole) and the first order autoregressive (AR1) covariance structure was chosen as the best fit for modeling the covariance among the repeated samples. For the three analyses, residuals were plotted and analyzed for normal distribution visually and via the $\mathrm{W}$ statistic (W) of the Shapiro-Wilk Test. In situations when interactions were significant, simple effects (main effect of each factor evaluated within each level) were tested using the SAS "slice" option. For significant simple effects, a post-hoc mean separation was performed using Tukey-Kramer's honestly significant difference (HSD) test. A fourth analysis tested the effect of genotype and plant part on the percentage of vessels occluded by gums and was performed by running one-way nonparametric Kruskal-Wallis tests.

To assess the reliability of the leaf scorch, CMI, and occlusion measurements for determining field resistance, the following procedure was performed. A quantitative measure of past field performance was made by assigning a field PD status rank to each genotype (Table 1). The field-resistant genotypes Blanc Du Bois, Roucaneuf, Tampa, Zehnder 71-50-1, and D8909-15 were given a field PD status rank of 1 . The moderately field-resistant D'Arpa was given a rank of 2 . The moderately field-susceptible Chenin blanc was given a rank of 3 and the field-susceptible Chardonnay was given a rank of 4 . The leaf scorch, CMI, tylose, and gum occlusion measurements of each genotype were plotted onto their respective field PD status rank and correlation was measured with the $R^{2}$ regression coefficient.

\section{RESULTS}

Leaf scorch. Representative examples of the leaf scorching phenotype at 12 weeks post- $X$. fastidiosa and water inoculation in the field-susceptible genotypes Chardonnay and Chenin blanc and the field-resistant genotypes Roucaneuf and Tampa are shown in Figure 2. Leaf scorch scores were taken from the replicates of each genotype and, to achieve normally distributed residuals in the data set, 4 outliers of 63 data points were removed $(\mathrm{W}=$ $0.974, P=0.2248$ ). To calculate the combined effect of genotype and inoculation on leaf scorch values, the interaction of these two main factors was tested. The inoculation-genotype interaction was significant $(P<0.0001)$; therefore, to measure the effect of genotype separately within the two inoculation levels (water and $X$. fastidiosa), these simple effects were tested using the SAS slice option. The effect of genotype was significant $(P<0.0001)$ within both $X$. fastidiosa-inoculated and water-inoculated plants.

The mean percentage of leaf scorch on the water-inoculated genotypes were $0,5,7,8,8,13,20$, and 90\% for D8909-15, Roucaneuf, Chardonnay, Chenin blanc, Tampa, Zehnder 71-50-1, Blanc Du Bois, and D'Arpa, respectively. In a statistical post-hoc analysis among the water-inoculated plants, only D'Arpa was significantly different $(P<0.0001 \mathrm{HSD})$. No $X$. fastidiosa were detected within samples taken from the control replicates of D'Arpa (data not shown). Therefore, leaf scorch on D'Arpa was most likely due to poor adaptation to the greenhouse conditions. Mean leaf scorch area on all of the water-inoculated plants was under 20\%. When excluding the genotype D'Arpa, background leaf scorch area was less than $10 \%$.

An analysis was run with data from $X$. fastidiosa-inoculated plants, nesting genotype within field PD status. Both field PD status and genotype (nested within field PD status) were signifi- 
cant $(P<0.0001)$. The susceptible status had a mean leaf scorch percentage of $80 \%$ whereas the resistant status had a mean leaf scorch percentage of $48 \%$. Mean percent leaf scorch values of each genotype are presented in Table 2. D'Arpa had a high mean percent leaf scorch $(91 \%)$ similar to that of its water-inoculated controls. The remaining resistant genotypes had mean leaf scorch percentages considerably higher than their respective waterinoculated controls, ranging from $26 \%$ for Blanc Du Bois to $57 \%$ for Zehnder 71-50-1, indicating that the classic leaf scorch symptom can be extensive even in resistant genotypes (Fig. 2G and H). Among the individual genotypes, only Blanc Du Bois, Roucaneuf, and D8909-15 were significantly different from Chardonnay $(P=$ $0.0003,0.0021$, and $0.0031 \mathrm{HSD}$, respectively) (Table 2). The remaining resistant genotypes, Tampa, Zehnder 71-50-1, and
D'Arpa, were not significantly different from Chardonnay $(P=$ $0.3049,0.6949$, and $0.6233 \mathrm{HSD}$, respectively). The regression of mean percent leaf scorch values of each genotype on to their corresponding field PD status rank showed only a moderate correlation $\left(R^{2}=0.51\right)$ to each other.

Cane maturation. Representative examples of the irregular cane maturation phenotype at 12 weeks post- $X$. fastidiosa and water inoculation in the susceptible Chardonnay and Chenin blanc and the resistant Roucaneuf and Tampa are shown in Figure 3. CMI scores were taken from the replicates of each genotype and, to achieve normally distributed residuals in the data set, 3 outliers of 63 data points were removed ( $\mathrm{W}=0.960, P=0.05$ ). To calculate the combined effect of genotype and inoculation on CMI scores, the interaction of these factors was tested. The inter-
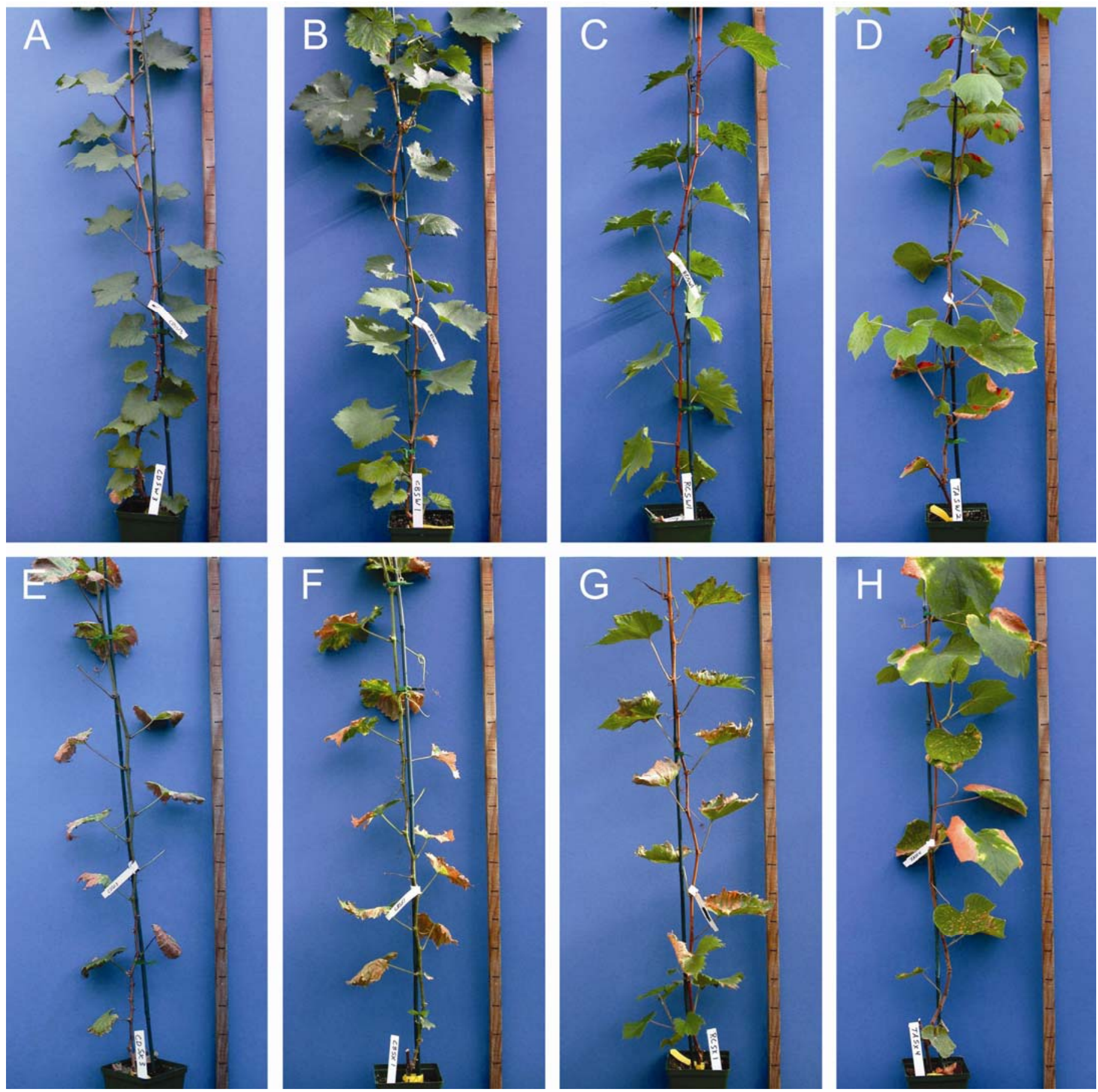

Fig. 2. Leaf scorch symptoms at 12 weeks post- A to D, water inoculation and $\mathbf{E}$ to $\mathbf{H}$, Xylella fastidiosa inoculation. Genotypes $\mathbf{A}$ and $\mathbf{E}$, Chardonnay and $\mathbf{B}$ and $\mathbf{F}$, Chenin blanc are field susceptible; genotypes $\mathbf{C}$ and $\mathbf{G}$, Roucaneuf and $\mathbf{D}$ and $\mathbf{H}$, Tampa are field resistant. 
TABLE 2. Mean percentage leaf scorch for each genotype 12 weeks post-Xylella fastidiosa inoculation

\begin{tabular}{|c|c|c|c|c|c|}
\hline Genotype & Rep no. & Mean leaf scorch $(\%)^{\mathrm{y}}$ & SE & Minimum leaf scorch (\%) & Maximum leaf scorch (\%) \\
\hline Blanc Du Bois & 5 & $25.5 \mathrm{a}$ & 4.9 & 10.0 & 40.0 \\
\hline Roucaneuf & 4 & $30.0 \mathrm{a}$ & 2.3 & 25.0 & 35.0 \\
\hline D8909-15 & 5 & $34.0 \mathrm{a}$ & 7.6 & 17.5 & 62.5 \\
\hline Tampa & 5 & $53.0 \mathrm{ab}$ & 6.9 & 35.0 & 67.5 \\
\hline Zehnder71-50-1 & 3 & $56.7 \mathrm{ab}$ & 12.1 & 32.5 & 70.0 \\
\hline Chardonnayz & 5 & $74.5 \mathrm{bc}$ & 7.4 & 60.0 & 100.0 \\
\hline Chenin blanc ${ }^{\mathrm{z}}$ & 3 & $88.3 \mathrm{c}$ & 8.2 & 72.5 & 100.0 \\
\hline D'Arpa & 5 & $91.0 \mathrm{c}$ & 5.6 & 75.0 & 100.0 \\
\hline
\end{tabular}

${ }^{y}$ Results followed by different letters differ significantly $(P<0.05)$ using Tukey-Kramer's honestly significant difference.

${ }^{\mathrm{z}}$ Field-susceptible genotypes.
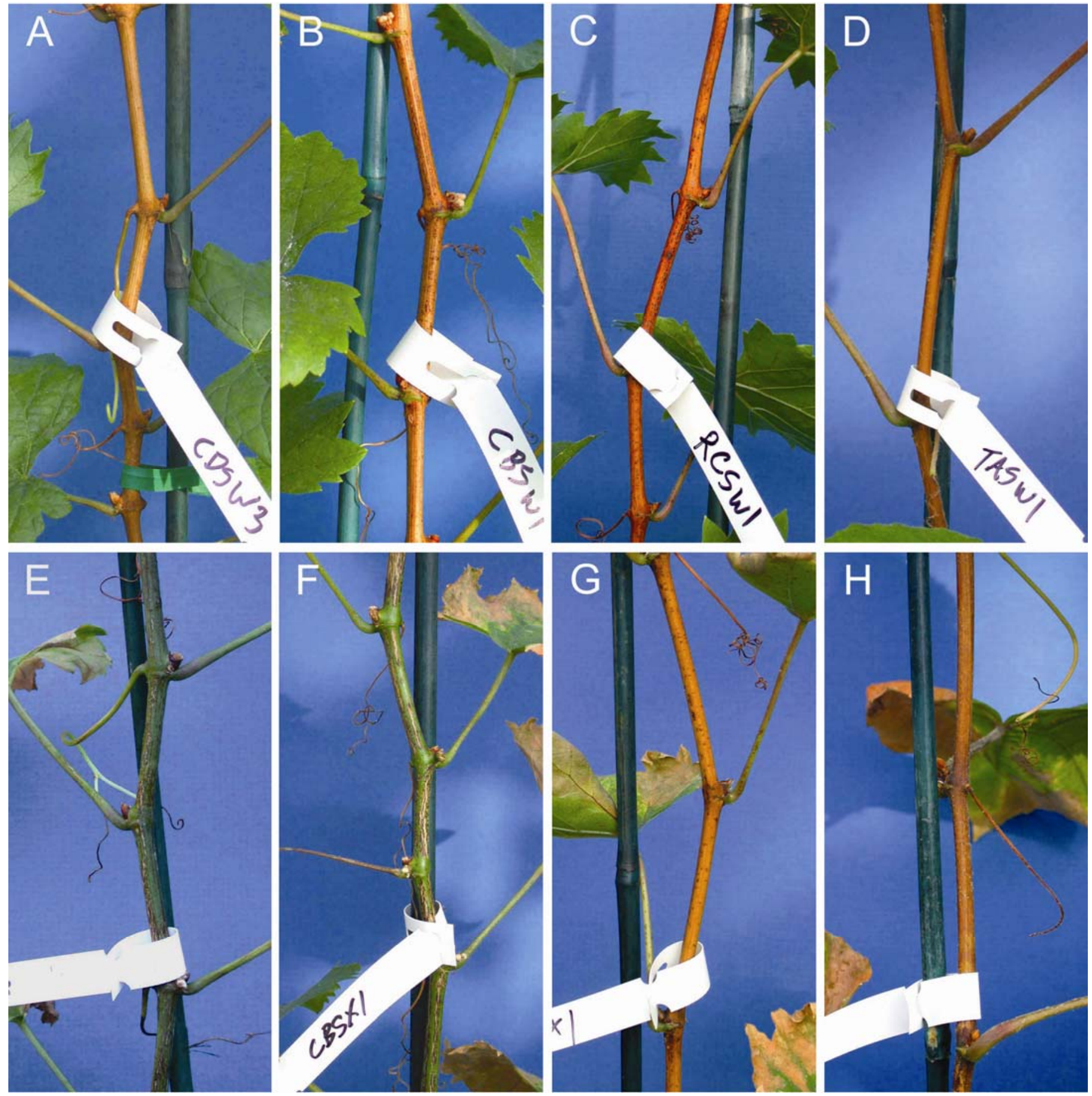

Fig. 3. Cane maturation symptoms at 12 weeks post- A to D, water inoculation and $\mathbf{E}$ to $\mathbf{H}$, Xylella fastidiosa inoculation. Genotypes $\mathbf{A}$ and $\mathbf{E}$, Chardonnay and $\mathbf{B}$ and $\mathbf{F}$, Chenin blanc are field susceptible; genotypes $\mathbf{C}$ and $\mathbf{G}$, Roucaneuf and $\mathbf{D}$ and $\mathbf{H}$, Tampa are field resistant. 
action of inoculation-genotype was significant $(P<0.0001)$; therefore, the simple effect of genotype within the two inoculation levels (water and $X$. fastidiosa) was tested using the SAS slice option. In water-inoculated plants, the effect of genotype on CMI scores was not significant $(P=0.950)$. The mean CMI scores of the water-inoculated plants was $0.0,0.3,0.3,0.5,0.5,0.5,0.7$, and 0.7 (on a 0-to-6 scale) for D8909-15, Chardonnay, Zehnder 71-50-1, Roucaneuf, Tampa, Chenin blanc, Blanc Du Bois, and D'Arpa, respectively.

Among the $X$. fastidiosa-inoculated plants, the simple effects of field PD status and genotype (nested within field PD status) were both significant $(P<0.0001$ and $P=0.0257$, respectively). Consistent with this initial $F$ test, a statistical post-hoc analysis showed that the susceptible and resistant status were significantly different from each other $(P<0.0001$ HSD), with the resistant field PD status having a mean CMI number of 0.9 and the susceptible field PD status a mean CMI score of 5.1 (on a 0-to-6 scale). Each of the resistant genotypes was significantly different from the two susceptible genotypes $(P<0.001 \mathrm{HSD})$. Resistant genotype CMI scores ranged from 0.4 for D8909-15 to 2.3 for moderately field-resistant D'Arpa (Table 3), and these were the only resistant genotypes significantly different from each other $(P=0.038$ HSD). D'Arpa canes were mostly tan or pale green and had very little necrotic tissue at the shoot apex; thus, its higher CMI score relative to the other resistant genotypes was predominantly due to a higher percentage of irregular cane maturation. The regression of CMI scores on field PD status rank of each genotype yielded a regression coefficient of $R^{2}=0.91$, indicating that the CMI scoring method is a highly reliable predictor of field PD resistance.

Tylose occlusions. Representative tylose and gum occlusions in petiole vessels as observed 12 weeks post- $X$. fastidiosa inoculation are shown in Figure 4. For all genotypes and in both plant parts, the majority of the vessels occluded were occluded by tyloses. Tyloses were counted in cross-sections of petioles and stems following dissection and visual inspection under magnification. Less than $1 \%$ of vessels were occluded with tyloses in the water-inoculated controls; therefore, an analysis was run only on the $X$. fastidiosa-inoculated plants. To achieve normally distributed residuals in the analysis of the tylose occlusion data set, 4 outliers of 73 data points were removed $(\mathrm{W}=0.99, P=0.8012)$. In order to see the effects of PD status and genotype within the two plant part levels (petiole and stem), these simple effects were evaluated using the slice option. No significant effect on tylose formation within petioles by PD status or genotype was found $(P=0.3857$ and 0.1879 , respectively). Petioles of the resistant PD status had a mean percentage of vessels occluded by tyloses of 36 compared with $39 \%$ of the susceptible PD status. Among the individual genotypes, the mean percentage of tylose occlusions in petioles ranged from a low of $27 \%$ in Zehnder 71-50-1 to $41 \%$ in D8909-15.

Within internode samples, the effects of field PD status and genotype on tylose occlusions were significant $(P<0.0233$ and 0.0001 , respectively). The resistant and susceptible PD status had tylose occluded internode vessels at a mean percentage of 20 and
$26 \%$, respectively. Among the individual genotypes, the mean percentage of tylose-occluded vessels in internode samples ranged from a low of $4 \%$ in D8909-15 to a high of 36\% in Blanc Du Bois (Table 4). Of the resistant genotypes, only D8909-15 had a significantly lower percentage of vessels occluded by tyloses than both of the susceptible genotypes $(P=0.0092$ and $P<0.0001$ HSD, respectively). The regression of internode tyloses on field PD status rank yielded a low correlation $\left(R^{2}=0.13\right)$, indicating that the use of tylose occlusion measurements in stem vessels is a poor predictor of field PD resistance. An obvious trend of a low percentage of vessels occluded by tyloses within internodes relative to the higher percentage of vessels occluded in petioles was evident in all genotypes (Table 4). The effect of plant part on tylose formation proved to be significant within either PD status backgrounds $(P<0.0001$ and $P=0.0017$, respectively).

Gum occlusions. In the water-inoculated controls, less than $1 \%$ of vessels were occluded with gums; therefore, an analysis was run only on the $X$. fastidiosa-inoculated plants. Among the $X$. fastidiosa-inoculated plants, the mean percentages of vessels occluded by gums never exceeded $10 \%$ in any sample. The low level of gum occlusions resulted in a data set where assumptions of normally distributed residuals and homogeneous variances could not be met. As such, the data were analyzed by running one-way nonparametric analysis of variance. The effect of genotype on gum occlusions was significant $(P=0.0101)$ when analyzed within petioles, but not significant when analyzed within internodes $(P=0.0624)$. The percentage of internode vessels occluded by gums was $0,0,0,0,0.2,0.2,0.6$, and $1 \%$ and the percentage of petiole vessels occluded by gums was $2.2,5.8,1.4$, $3.2,1.5,3.7,9.3$, and $8.2 \%$ for D8909-15, Roucaneuf, Tampa,

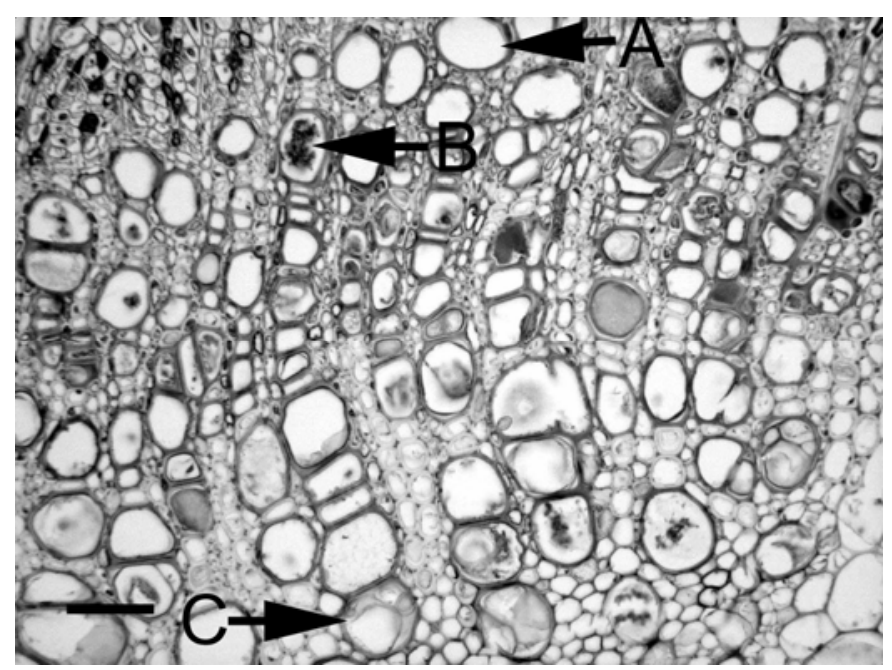

Fig. 4. A, Nonoccluded vessel, B, gum-occluded vessel, and C, tylose-occluded vessel in a cross section of a petiole from a susceptible Xylella fastidiosainoculated grapevine. Light micrograph was taken 12 weeks postinoculation at $\times 200$ magnification. Scale bar $=50 \mu \mathrm{m}$.

TABLE 3. Mean cane maturation index (CMI) score (on a 0-to-6 scale) for each genotype 12 weeks post-Xylella fastidiosa inoculation

\begin{tabular}{lcclcc}
\hline Genotype & Rep no. & Mean CMI score & SE & Minimum CMI score & Maximum CMI score \\
\hline D8909-15 & 5 & $0.4 \mathrm{a}$ & 0.2 & 0.0 & 0.0 \\
Roucaneuf & 4 & $0.5 \mathrm{ab}$ & 0.3 & 0.0 & 1.0 \\
Tampa & 5 & $0.6 \mathrm{ab}$ & 0.4 & 0.0 & 2.0 \\
Zehnder 71-50-1 & 5 & $0.6 \mathrm{ab}$ & 0.4 & 0.0 & 1.0 \\
Blanc Du Bois & 5 & $1.4 \mathrm{ab}$ & 0.5 & 4.0 & 3.0 \\
D'Arpa & 4 & $2.3 \mathrm{~b}$ & 0.5 & 3.0 & 6.0 \\
Chardonnay $^{\mathrm{z}}$ & 5 & $5.0 \mathrm{c}$ & 0.3 & 5.0 & 6.0 \\
Chenin blanc $^{\mathrm{z}}$ & 4 & $5.3 \mathrm{c}$ & 0.3 & 0 & \\
\hline
\end{tabular}

${ }^{y}$ Results followed by different letters differ significantly $(P<0.05)$ using Tukey-Kramer's honestly significant difference.

${ }^{\mathrm{z}}$ Field-susceptible genotypes. 
Zehnder 71-50-1, Blanc Du Bois, D'Arpa, Chardonnay, and Chenin blanc, respectively. A trend among the individual genotypes of low percentage of internode vessels occluded by gums relative to a higher percentage of petiole vessels occluded by gums was evident, and this trend was similar to that seen with tylose formation. The effect of plant part on gum formation proved highly significant, just as it did with tylose formation when evaluated within either the field-resistant or field-susceptible PD status levels $(P<0.0001$ and $P=0.0013$, respectively $)$.

\section{DISCUSSION}

This study has more precisely characterized the PD resistance phenotype of a diverse group of Vitis bunch grape genotypes under greenhouse conditions. In general, when challenged by $X$. fastidiosa, genotypes with high field PD resistance have canes that mature normally over an 8- to 12 -week period with no abnormal discoloration or necrosis of the cork tissues. The CMI, a measure of cork development, correlated highly to reported field PD performance $\left(R^{2}=0.91\right)$ and can measure disease progression effectively on a quantitative scale. In contrast, the percentage of leaf scorching had only a moderate correlation $\left(R^{2}=0.51\right)$ to field PD performance in this study.

The reactions of the genotypes to $X$. fastidiosa infection and measurements of disease manifestation also lend insight into the plant-pathogen interaction. Resistant genotypes were shown to have significantly higher levels of the classic leaf scorch symptom relative to water-inoculated controls, with up to $57 \%$ for genotypes with high field resistance. This result suggests that systemic infection can occur in all evaluated Vitis genotypes (under artificial inoculation and the presented greenhouse conditions) regardless of their field reactions. In a companion study (23), much of the scorching in the same set of resistant genotypes was associated with $X$. fastidiosa infection and high bacterial populations in the leaves and petioles.

Our phenotypic data on leaf scorch symptoms under greenhouse conditions are consistent with several field studies where leaf scorching and marginal necrosis have been documented in PD-resistant genotypes $(16,18,21,25,26)$. These studies were not conclusive evidence that systemic $X$. fastidiosa infection occurs in PD-resistant genotypes because inoculations by native sharpshooter vectors likely occurred at many points on the field-grown grapevines. One evaluation compared the field-susceptible V. labrusca 'Schuyler' to the field-resistant $M$. rotundifolia 'Carlos' in a Leesburg, FL vineyard with heavy PD pressure, and both cultivars had leaf marginal necrosis each year of the study (21). In addition, stem dieback symptoms (which are the ultimate manifestation of irregular cane maturation) also were measured and found more common in the susceptible Schuyler, which is in agreement with our greenhouse CMI evaluations.

The resistant genotypes, as a group, had significantly lower levels of leaf scorch than did the field-susceptible genotypes, making the classic leaf scorch phenotype a potentially viable choice for greenhouse PD resistance assessments. However, the primary problem with leaf scorch scoring is the elevated level of false positive errors (scoring a susceptible plant as resistant). Leaf scorch due to $X$. fastidiosa infection of leaves of field-resistant genotypes would confound results in a PD resistance evaluation, as would leaf scorch due to background environmental stresses. For example, the genotype D'Arpa generally was less well adapted to greenhouse conditions, leading to higher rates of leaf scorch and defoliation, and confounding PD resistance evaluations using D'Arpa or a similar genotype. The greenhouse environment can avoid most of the biotic and environmental effects that cause leaf scorching, but such effects often cannot be completely eliminated.

The irregular cane maturation phenotype of susceptible grapevines is a more unique PD symptom following artificial inoculation under greenhouse conditions than is leaf scorching. Irregular cork maturation and necrosis rarely are caused by other biotic or environmental stresses, as was demonstrated by the low background CMI scores of the water control plants in this study. The increased specificity of the CMI scores greatly lowers the chances for false positive errors in PD resistance evaluations. However, a potential problem with the use of CMI scores is false-negative errors (scoring a resistant plant as susceptible). If the plants are inoculated at too late a stage, even susceptible grapevines will initiate normal cork development before $X$. fastidiosa infection can influence cane maturation (data not shown). In order to reduce the rate of errors with the CMI evaluation method, it is critical that plants be inoculated at an early stage of growth, at least 1 month before the normal cane maturation process would typically begin. Another factor that can influence the cane maturation phenotype is irrigation. In a study comparing PD symptoms under normal and water stress conditions for greenhouse-grown grapevines, it was shown that the green island or irregular cane maturation symptom did not manifest in $X$. fastidiosa-infected Chardonnay under moderate water deficits (37). Proper irrigation management must be achieved when using the cane maturation phenotype for PD resistance evaluations.

Researchers have suggested that the occlusions associated with symptomatic grapevines may be produced by the plant in order to arrest $X$. fastidiosa infection or may be associated with the bacteria and facilitate infection $(8,22,27)$. In this study, a strong correlation between field resistance status and types or numbers of occlusions was not identified. However, results in a time-course

TABLE 4. Mean percentage of xylem vessels occluded by tyloses for each genotype 12 weeks post-Xylella fastidiosa inoculation

\begin{tabular}{|c|c|c|c|c|c|c|}
\hline Genotype & Plant part & Rep no. & $\begin{array}{c}\text { Mean vessels occluded } \\
\text { by tyloses }(\%)\end{array}$ & SE & $\begin{array}{c}\text { Minimum vessels } \\
\text { occluded by tyloses }(\%)\end{array}$ & $\begin{array}{c}\text { Maximum vessels } \\
\text { occluded by tyloses }(\%)\end{array}$ \\
\hline Blanc Du Bois & Internode & 5 & 36.3 & 2.7 & 29.2 & 45.4 \\
\hline Blanc Du Bois & Petiole & 5 & 37.6 & 2.5 & 32.7 & 47.0 \\
\hline Chardonnayz & Internode & 5 & 29.5 & 0.7 & 27.5 & 31.1 \\
\hline Chardonnay $^{\mathrm{z}}$ & Petiole & 5 & 38.6 & 2.8 & 31.0 & 46.0 \\
\hline Chenin blanc ${ }^{\mathrm{z}}$ & Internode & 4 & 21.5 & 3.0 & 16.0 & 27.4 \\
\hline Chenin blanc ${ }^{\mathrm{z}}$ & Petiole & 3 & 38.9 & 4.2 & 33.6 & 47.3 \\
\hline D’Arpa & Internode & 5 & 20.3 & 1.0 & 18.0 & 23.1 \\
\hline D’Arpa & Petiole & 3 & 34.5 & 7.8 & 19.0 & 42.6 \\
\hline D8909-15 & Internode & 3 & 4.0 & 1.3 & 2.0 & 6.5 \\
\hline D8909-15 & Petiole & 5 & 40.6 & 4.2 & 31.8 & 54.0 \\
\hline Roucaneuf & Internode & 4 & 13.5 & 4.0 & 2.0 & 20.8 \\
\hline Roucaneuf & Petiole & 2 & 37.8 & 1.3 & 36.5 & 39.0 \\
\hline Tampa & Internode & 5 & 15.9 & 4.4 & 2.0 & 27.4 \\
\hline Tampa & Petiole & 5 & 39.1 & 2.9 & 30.2 & 46.1 \\
\hline Zehnder71-50-1 & Internode & 5 & 22.9 & 4.3 & 11.9 & 34.6 \\
\hline Zehnder 71-50-1 & Petiole & 4 & 26.9 & 4.7 & 15.8 & 36.4 \\
\hline
\end{tabular}

${ }^{\mathrm{z}}$ Field-susceptible genotypes. 
study (36) indicate that tylose formation develops well after $X$. fastidiosa colonization and it is possible that the percentage of internode vessels occluded within the resistant genotypes will stabilize, whereas they continue to develop in susceptible genotypes. Further research utilizing time-course studies of $X$. fastidiosa infection within resistant and susceptible backgrounds is needed.

The use of CMI scores for PD resistance evaluations is highly feasible due to its ease of use and the high correlation to field performance. The 5- to 7-month duration of the greenhouse evaluation is considerably shorter and, therefore, more efficient than the multiyear field studies previously conducted and should facilitate the development of new PD-resistant cultivars.

\section{ACKNOWLEDGMENTS}

Funding for this research was provided by the California Department of Food and Agriculture PD Board, the American Vineyard Foundation, the North Coast PD Task Force, and the Louis P. Martini Endowed Chair Funds and is gratefully acknowledged. Scholarship support to A. F. Krivanek from the following sources is also gratefully acknowledged: the American Society for Enology and Viticulture, the American Wine Society, the James Beard Foundation, and the André Tchelistcheff Foundation. We thank B. Kirkpatrick, D. Skinner, and the Phytopathology reviewers for helpful input on the revisions of this manuscript; A. Tenscher for assistance in selection of the evaluated genotypes; and A. Skinner for help with plant care in the field and greenhouse.

\section{LITERATURE CITED}

1. Amorim, L., Bergamin Filho, A., Palazzo, D. A., Bassanezi, R. B., Godoy, C. V., and Torres, G. A. M. 1993. Citrus variegation chlorosis: A diagrammatic scale for the evaluation of the severity of the disease. Fitopatol. Bras. 18:174-180.

2. Berisha, B., Chen, Y. D., Zhang, G. Y., Xu, B. Y., and Chen, T. A. 1998. Isolation of Pierce's disease bacteria from grapevines in Europe. Eur. J. Plant Pathol. 104:427-433.

3. Butler, O. 1910. Observations on the California vine disease. Mem. Torrey Bot. Club 14:111-153.

4. Davis, M. J., French, W. J., and Schaad, N. W. 1981. Axenic culture of the bacteria associated with phony disease of peach and plum leaf scald. Curr. Microbiol. 6:309-314.

5. Davis, M. J., Purcell, A. H., and Thomson, S. V. 1980. Isolation media for the Pierce's disease bacterium. Phytopathology 70:425-429.

6. Esau, K. 1948. Anatomic effects of the viruses of Pierce's disease and phony peach. Hilgardia 18:423-482.

7. Freitag, J. H. 1951. Host range of the Pierce's disease virus of grapes as determined by insect transmission. Phytopathology 41:920-934.

8. Fry, S. M., and Milholland, R. D. 1990. Response of resistant tolerant and susceptible grapevine tissues to invasion by the Pierce's disease bacterium Xylella fastidiosa. Phytopathology 80:66-69.

9. Galet, P. 1988. Cepages et Vignobles de France, tome 1. Les Vignes Americaines. 2nd ed. Charles Déhan Press, Montpellier, France.

10. Goodwin, P. H., and Purcell, A. H. 1992. Pierce's disease. Pages 76-84 in: Grape Pest Management. D. P. Flaherty, ed. University of California, Division of Agriculture and Natural Resources, Oakland.

11. Halbrooks, M. C., and Mortensen, J. A. 1989. Origin and significance of Florida hybrid bunch grapes and rootstocks. Hortscience 24:546-550.

12. Hewitt, W. B. 1958. The probable home of Pierce's disease virus. Plant Dis. Rep. 42:211-214.

13. Hewitt, W. B., Frazier, N. W., and Houston, B. R. 1942. Pierce's disease of grapevines. Calif. Agric. Exp. Stn. Circ. 353:1-32.
14. Hopkins, D. L. 1980. Use of pin-prick inoculation technique demonstrates variability in virulence of Pierce's disease bacterium. Pages 177-180 in: Proc. VIIth Int. Conf. Viruses Grapevines (ICVG), Niagara Falls, Canada.

15. Hopkins, D. L. 1981. Seasonal concentration of the Pierce's disease bacterium in grapevine Vitis labrusca cultivar Schuyler stems, petioles and leaf veins. Phytopathology 71:415-418.

16. Hopkins, D. L. 1984. Variability of virulence in grapevine among isolates of the Pierce's disease bacterium. Phytopathology 74:1395-1398.

17. Hopkins, D. L. 1989. Xylella fastidiosa xylem-limited bacterial pathogen of plants. Annu. Rev. Phytopathol. 27:271-290.

18. Hopkins, D. L., Mollenhauer, H. H., and Mortensen, J. A. 1974. Tolerance to Pierce's disease and the associated rickettsia-like bacterium in muscadine grape. J. Am. Soc. Hortic. Sci. 99:436-439.

19. Hopkins, D. L., and Purcell, A. H. 2002. Xylella fastidiosa: Cause of Pierce's disease of grapevine and other emergent diseases. Plant Dis. 86:1056-1066

20. Hopkins, D. L., and Thompson, C. M. 1981. Multiplication of virulent and avirulent Pierce's disease bacterial isolates in grapevine tissue. Pages 225-234 in: Proc. Vth Int. Conf. Plant Pathol. Bacteriol. Cali, Colombia.

21. Hopkins, D. L., and Thompson, C. M. 1984. Seasonal concentration of the Pierce's disease bacterium in 'Carlos' and 'Welder' muscadine grapes compared with 'Schuyler' bunch grape. Hortscience 19:419-420.

22. Huang, P. Y., Milholland, R. D., and Daykin, M. E. 1986. Structural and morphological changes associated with the Pierce's disease bacterium in bunch and Muscadine grape tissues. Phytopathology 76:1232-1238.

23. Krivanek, A. F., and Walker, M. A. 20054. Vitis resistance to Pierce's disease is characterized by differential Xylella fastidiosa populations in stems and leaves. Phytopathology 95:44-52.

24. Loomis, N. H. 1958. Performance of Vitis species in the south as an indication of their relative resistance to Pierce's disease. Plant Dis. Rep. 42:833-836

25. Lu, J., and Cousins, P. 2003. Field evaluation of grape rootstock response to natural infection by Pierce's disease. Pages 19-22 in: 2003 Pierce's Disease Res. Symp. Coronado, CA.

26. Milholland, R. D., Huang, P. Y., Clayton, C. N., and Jones, R. K. 1981. Pierce's disease on muscadine grapes in North Carolina USA. Plant Dis. 65:73-74.

27. Mollenhauer, H. H., and Hopkins, D. L. 1976. Xylem morphology of Pierce's disease infected grapevines with different levels of tolerance. Physiol. Plant Pathol. 9:95-100.

28. Mortensen, J. A. 1988. Blanc Du Bois grape. Hortscience 23:418-419.

29. Mortensen, J. A., and Stover, L. H. 1982. Tampa grape rootstock. Hortscience 17:273-274.

30. Mortensen, J. A., Stover, L. H., and Balerdi, C. F. 1977. Sources of resistance to Pierce's disease in Vitis. J. Am. Soc. Hortic. Sci. 102:695-697.

31. Pratt, C. 1974. Vegetative anatomy of cultivated grapes-A review. Am. J. Enol. Vitic. 25:131-150.

32. Purcell, A. H. 1974. Spatial patterns of Pierce's disease in the Napa Valley. Am. J. Enol. Vitic. 25:162-167.

33. Purcell, A. H. 1981. Vector preference and inoculation efficiency as components of resistance to Pierce's disease in European grape cultivars. Phytopathology 71:429-435.

34. Raju, B. C., and Goheen, A. C. 1981. Relative sensitivity of selected grapevine cultivars to Pierce's disease bacterial inoculations. Am. J. Enol. Vitic. 32:155-158.

35. Ruzin, S. E. 1999. Plant Microtechnique and Microscopy. Oxford University Press, New York.

36. Stevenson, J. F., Matthews, M. A., Greve, L. C., Labavitch, J. M., and Rost, T. L. Grapevine susceptibility to Pierce's disease II: The progression of anatomical symptoms. Am. J. Enol. Vitic. 55:238-245.

37. Thorne, E. T., Labavitch, J. M., Rost, T. L., and Matthews, M. A. 2003. Pierce's disease symptoms: Comparison with symptoms of water deficit and the impact of water stress. Page poster in: 2003 Pierce's Disease Res. Symp. Coronado, CA. 